\title{
The expression and role of peroxisome proliferator-activated receptor $\alpha$ in atherosclerosis
}

\author{
WEN GAO ${ }^{1 *}$, WEI XU ${ }^{2 *}$ and HONGLI LI ${ }^{1}$ \\ ${ }^{1}$ Department of Cardiology, Shanghai First People's Hospital, College of Medicine, Shanghai Jiaotong University, \\ Shanghai 200080; ${ }^{2}$ Department of Interventional Radiology, the Second Affiliated Hospital \\ of Harbin Medical University, Harbin, Heilongjiang 150086, P.R. China
}

Received October 2, 2012; Accepted January 11, 2013

DOI: $10.3892 / \mathrm{mmr} .2013 .1282$

\begin{abstract}
Peroxisome proliferator-activated receptor $\alpha$ (PPAR- $\alpha$ ) has been detected in the liver, kidney, heart and skeletal muscle. The expression and mechanism of PPAR- $\alpha$ in atherosclerosis remains unclear. The present study was undertaken in order to examine the expression and role of PPAR- $\alpha$ in programmed atherosclerosis induced by a high-fat diet and balloon-injury in rabbits. Rabbits were randomly divided into 3 groups: control, high-fat diet and high-fat diet + balloon-injury groups. The high-fat diet and high-fat diet + balloon-injury groups were further divided into 6-, 8and 10-week groups. Real-time quantitative PCR analysis was used to detect PPAR- $\alpha$ mRNA and immunohistochemistry (IHC) and western blot analysis were used to examine PPAR- $\alpha$ protein expression. Tumor necrosis factor (TNF)- $\alpha$, interleukin (IL)-10 and P-selectin levels in the rabbits were measured by enzyme-linked immunosorbent assay (ELISA). In the high-fat or high-fat diet + balloon-injury groups, the vascular thickness was markedly higher than in the control group $(\mathrm{P}<0.01)$. PPAR- $\alpha$ protein and mRNA were significantly increased in the high-fat diet group as compared with the control group $(\mathrm{P}<0.01)$. Furthermore, there were marked changes from 6 to 10 weeks in the high-fat diet group $(\mathrm{P}<0.01)$. Compared with the control group, PPAR- $\alpha$ protein and mRNA were increased in the highfat diet + balloon-injury group $(\mathrm{P}<0.01)$. There were significant differences of PPAR- $\alpha$ protein and mRNA at various time points in the high-fat diet + balloon-injury group, as shown by real-time quantitative PCR and IHC $(\mathrm{P}<0.01)$. As shown by western blotting, there were no differences between the highfat diet + balloon-injury 8 - and 10 -week groups ( $P>0.05)$. In those arteries that were occluded by $\geq 60 \%$, PPAR- $\alpha$ expression
\end{abstract}

Correspondence to: Dr Hongli Li, Department of Cardiology, Shanghai First People's Hospital, College of Medicine, Shanghai Jiaotong University, 100 Haining Road, Shanghai 200080, P.R. China E-mail: hongli.li@yahoo.cn

*Contributed equally

Key words: peroxisome proliferator-activated receptor, PPAR- $\alpha$, inflammation, atherosclerosis was lower than that in the arteries which were occluded $<60 \%$ in the high-fat diet + balloon-injury 10-week group. In the highfat diet and high-fat diet + balloon-injury groups, the levels of IL-10, TNF- $\alpha$ and P-selectin were upregulated compared with the control group. However, from weeks 8 to 10 , TNF- $\alpha$ and P-selectin were decreased and IL-10 was still increased in the high-fat diet + balloon-injury group. The results of this study demonstrate that PPAR- $\alpha$ has preventive effects on atherosclerosis, which may be related to the regulation of inflammation.

\section{Introduction}

Acute cardiovascular events, including myocardial infarction, stroke and sudden death, which are induced by atherosclerosis, remain the principal causes of morbidity and mortality globally (1). Increasing epidemiological evidence implies that inflammation contributes to the development and progression of atherosclerotic disease. However, the underlying mechanisms of atherosclerosis are not clear. Recent data suggest that peroxisome proliferator-activated receptor $\alpha$ (PPAR- $\alpha$ ) has multiple effects, which may be beneficial to alleviate atherosclerotic lesions.

The evolving view of atherosclerosis as a metabolic complication has directed attention toward PPARs as nuclear receptors which have three subtypes, PPAR- $\alpha, \beta / \delta$ and $\gamma$ (2). PPAR- $\alpha$ has been detected in the liver, kidney, heart and skeletal muscle, since hepatocytes and smooth muscle cells, as well as macrophages and endothelial cells, are represented in these organs (3). PPAR- $\alpha$ regulates fatty acid catabolism and glucose homeostasis, while it inhibits cytokine-induced expression of vascular cell-adhesion molecule-1 (VCAM-1), intercellular adhesion molecule-1 (ICAM-1), C-reactive protein (CRP) and interleukin (IL)-6 as a result of potentially modulating inflammation (4-9). Among pro- and anti-inflammatory cytokines, tumor necrosis factor- $\alpha$ (TNF- $\alpha)$ and IL-10 play predominant roles in the formation of atherosclerosis (10). Another problem we focus on in this study is P-selectin. A previous study has shown that PPAR- $\alpha$ decreases platelet-derived growth factor-BB (PDGF-BB) via suppression of the PDGF-B gene in bone marrow megakaryocytes (11). PPAR- $\alpha$ expression and its modulatory role in inflammation have been studied under in vitro conditions. However, there are few data on the expression and role of PPAR- $\alpha$ in vivo. 
In order to further explore the expression and mechanisms of PPAR- $\alpha$, our objectives were to investigate: i) PPAR- $\alpha$ expression in an atherosclerotic rabbit model of plaque rupture induced by high-fat diet and balloon angioplasty; ii) the correlation between PPAR- $\alpha$ and TNF- $\alpha$, IL-10 and P-selectin.

\section{Materials and methods}

Animal model. Adult male New Zealand White rabbits weighing $2.0-2.5 \mathrm{~kg}$ (total $\mathrm{n}=35$ ), were purchased from Shanghai Experimental Animal Breeding Co., China. After adaptation to the environment for 1 week, New Zealand White rabbits were randomly divided into 7 groups: control $(n=5)$, high-fat diet 6-week $(n=5)$, high-fat diet 8-week $(n=5)$, highfat diet 10 -week $(n=5)$, high-fat diet + balloon-injury 6 -week $(n=5)$, high-fat diet + balloon-injury 8 -week $(n=5)$ and high-fat diet + balloon-injury 10 -week $(n=5)$. Rabbits were housed in a temperature-, humidity- and light-controlled room with free access to water. The control group was provided with a normal diet, the high-fat group received only the high-fat diet, and the high-fat diet + balloon-injury group received the high-fat diet and balloon-injury. The high-fat diet was composed of cholesterol (2\%), lard (10\%) and normal diet (88\%). All experiments were performed according to the Experimental Animal Center of Shanghai First People's Hospital (SYXK, Shanghai, 2009-0086) and Use Committee guidelines.

Preparation of balloon injury. Rabbits $(n=15)$ were allowed to adapt to the environment for one week, and then femoral artery balloon-injury was carried out after rabbits were fed a high-fat diet for 4 weeks. Animals were anesthetized with an intravenous infusion of ketamine $(0.5 \mathrm{ml} / \mathrm{kg}$ Ketalar; Parke-Davis, Detroit, MI, USA) and xylazine ( $0.25 \mathrm{ml} / \mathrm{kg}$ Rompun; Bayer, Leverkusen, Germany). Femoral artery deendothelialization was induced by a 3-F Fogarty balloon catheter (Baxter, Shanghai, China). Using ophthalmic scissors to cut a V-shaped small hole in the artery wall, a balloon catheter (1:15 diluted heparin saline infiltration) was inserted retrograde into the iliac artery to $\sim 15 \mathrm{~cm}$, with a connection to a $20-\mathrm{ml}$ injector, and $\sim 10 \mathrm{ml}$ air was injected ( $\sim 2 \mathrm{~atm})$ to fill the balloon. The balloon was slowly pulled back to the femoral artery, and then the stretch was repeated twice for $30 \mathrm{sec}$ each time at an interval of $1 \mathrm{~min}$, to ensure intimal injury. The catheter was removed, the proximal end was ligated and the distal end was sutured with subcutaneous tissue and skin. To prevent infection, penicillin sodium liquid was used to clean wounds, and 400,000 units of penicillin sodium was injected intramuscularly for 3 days after the surgery.

Two weeks following the initial injury, 5 rabbits were sacrificed from the high-fat diet + balloon-injury 6-week group. The high-fat diet + balloon-injury 8-week group and high-fat diet + balloon-injury 10 -week group were created using the same method.

Histological assessment of artery damage. The artery was sliced transversely, and a midventricular slice was fixed in $10 \%$ formalin for $24 \mathrm{~h}$, embedded in paraffin and cut into 3-mm sections for histological assessment. Paraffin sections were stained with hematoxylin and eosin. The percentage of vessel wall lumen occlusion was calculated as $1-[\mathrm{L}$ area/( $\mathrm{I}+\mathrm{L}$ area) x 100] (L, lumen; I, intima), as described by a previous

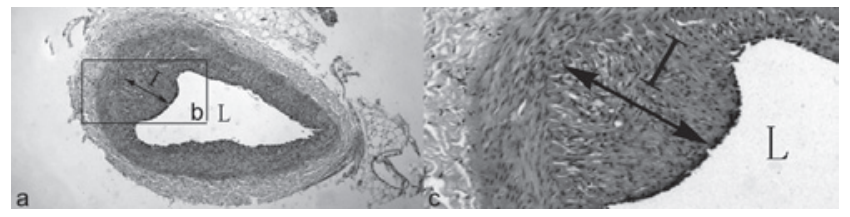

Figure 1. Percentage of vessel wall lumen occlusion was calculated as 1 - [L area/(I+L area) x 100]. L, lumen; Im, intima. Magnification, a is x50; c is $\mathrm{x} 400$ (c is higher magnification of $\mathrm{b}$ ).

study (12). The calculation method is shown in Figure 1. All measurements were made by an investigator blinded to the treatment and injury conditions.

Immunohistochemistry. Paraffin sections of the artery were deparaffinized, and endogenous peroxidase activity was inactivated with $3 \% \mathrm{H}_{2} \mathrm{O}_{2}$ for $10 \mathrm{~min}$. The primary antibody (mouse anti-rabbit PPAR- $\alpha$, no. NB300-537; Novus, Cambridge, UK) or normal blocking serum was added and incubated overnight. Biotin-conjugated goat anti-mouse immunoglobulin $\mathrm{G}$ (IgG) was used as the secondary antibody and incubated for $30 \mathrm{~min}$. An avidin-biotin enzyme reagent was sequentially added and incubated for $20 \mathrm{~min}$. A peroxidase substrate was added and incubated until the desired stain intensity developed. Finally, sections were covered with a glass coverslip and observed using a light microscope. The intensity of positive staining in tissue was analyzed by integrated optical density (IOD) using Image-Pro Plus software (IPP; Media Cybernetics, Rockville, MD, USA). Briefly, 4 x20 TIF-format images from five individual rabbits in each group were analyzed in a blinded manner. At the end of the analysis, the IOD and area were obtained, as well as the lumen area and internal elastic lamina area. The PPAR- $\alpha$ expression determined by immunohistochemistry (IHC) was expressed as (IOD/area) x 100 in accordance with a previous study (13).

Western blot analysis. Specimens were washed with ice-cold PBS and lysed for $20 \mathrm{~min}$ on ice with lysis buffer. Following lysis (no. SC-003; Invent, Lund, Sweden), the lysates were centrifuged for $4 \mathrm{~min}$ at $12,000 \mathrm{rpm}$, and the supernatants were collected in a fresh tube kept on ice. Protein concentrations in each sample were determined using a BCA assay. Total proteins $(100 \mu \mathrm{g})$ were mixed with loading buffer containing the anionic denaturing detergent sodium dodecyl sulfate (SDS), boiled for $5 \mathrm{~min}$, and then resolved by $10 \%$ SDS polyacrylamide gel electrophoresis. The proteins were transferred onto a PVDF membrane. After blocking the membrane in TBST containing non-fat milk for $1 \mathrm{~h}$ at $4^{\circ} \mathrm{C}$ under agitation, the membrane was washed three times in TBST and incubated for $2 \mathrm{~h}$ with anti-rabbit PPAR- $\alpha$ antibody (1:200 dilution, no. NB300-537; Novus) or GAPDH monoclonal antibody (1:200 dilution, no. 20028, Abmart Company, Shanghai, China). After washing three times in TBST, the membrane was incubated with HRP-conjugated goat antimouse $\operatorname{IgG}(1: 1,000)$ at room temperature for $1 \mathrm{~h}$ and then washed three times with TBST. Immunobands were detected using a streptavidin amplification reagent (no. WBKL SOO 50; Millipore, Billerica, MA, USA) according to the manufacturer's instructions. 


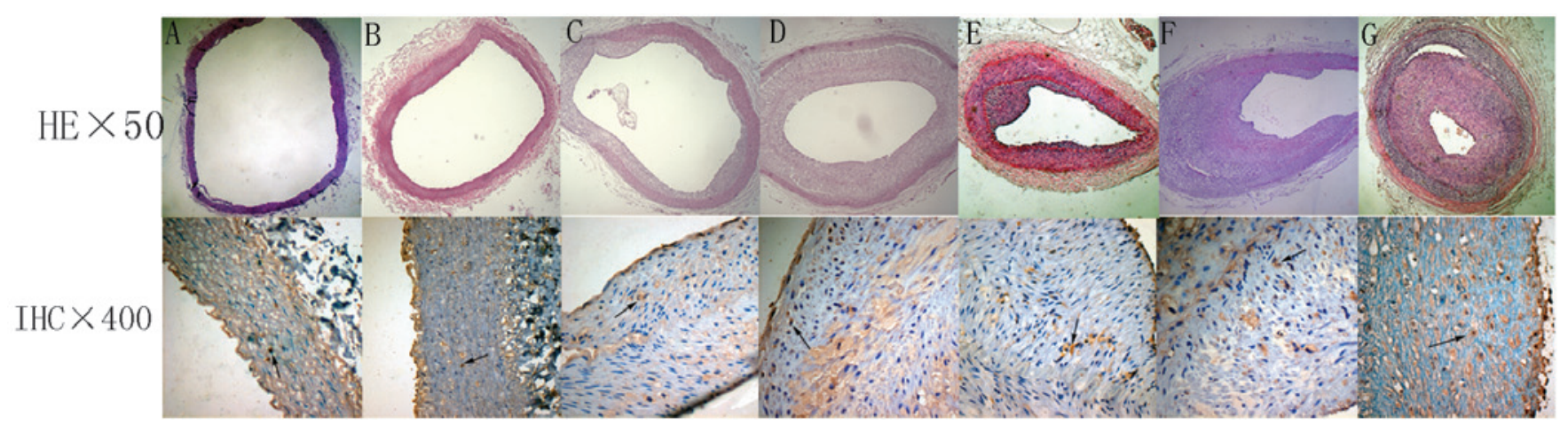

Figure 2. Microphotographs (H\&E x50 and IHC x400) of vasculature: (A) control group; (B) high-fat diet 6-week group; (C) high-fat diet 8-week group; (D) high-fat diet 10-week group (E) high-fat diet + balloon-injury 6-week group; (F) high-fat diet + balloon-injury 8-week group and (G) high-fat diet + balloon-injury 10 -week group. Arrows indicate positive staining.

RNA extraction and real-time PCR. Total RNA was extracted from the specimen arteries using TRIzol ${ }^{\circledR}$ reagent according to the manufacturer's instructions (Invitrogen, Carlsbad, CA, USA). Total RNA $(1 \mu \mathrm{g})$ was used as a template to produce cDNA using a reverse transcription kit (BioDev, Beijing, China). Real-time quantitative PCR was performed by monitoring the increase in fluorescence of the SYBR-Green dye using GreenMaster mix (Genaxxon BioScience, Ulm, Germany) according to the manufacturer's instructions. The primer sequences used to amplify PPAR- $\alpha$ were 5 '-gttccggtggcgttgat-3' (sense) and 5'-gcggtcgcatttgtc-3' (antisense). The primer sequences used to amplify GAPDH were 5 '-ccactttgtgaagctcatttcct-3' (sense) and 5'-tcgtcctcctctggtgctct-3' (antisense). PCR amplification was performed with $T a q$ polymerase for 32 cycles at $95^{\circ} \mathrm{C}$ for $45 \mathrm{sec}, 62^{\circ} \mathrm{C}$ for $30 \mathrm{sec}$, and $72^{\circ} \mathrm{C}$ for $1 \mathrm{~min}$ (for PPAR- $\alpha$ and GAPDH). The $2^{-\Delta \Delta C T}$ method was used to determine the relative change in PPAR- $\alpha$ and GAPDH gene expression. Values are expressed as relative quantities (RQ) compared with mRNA expression in the control group.

Enzyme-linked immunosorbent assay (ELISA) for TNF- $\alpha$, $I L-10$ and P-selectin. Fresh blood ( $3 \mathrm{ml})$ was taken from all animals via the femoral vein and samples were centrifuged at $3,000 \mathrm{rpm}$ for $10 \mathrm{~min}$ at $4^{\circ} \mathrm{C}$. The supernatant was stored in a clean centrifuge tube and frozen at $-20^{\circ} \mathrm{C}$. Concentrations of plasma TNF- $\alpha$, IL-10 and P-selectin were assayed with an ELISA kit (R\&D, Minneapolis, MN, USA) according to the manufacturer's instructions. The minimum detectable concentration of the kit was $<1.0 \mathrm{pg} / \mathrm{ml}$. The kit did not cross-react with other soluble structural analogs.

Statistical analysis. The data were analyzed using the program SPSS 11.5 for Windows. Quantitative data are presented as the means \pm SD. For comparison between multiple groups, data were analyzed by ANOVA and with the Student-NewmanKeuls post hoc analysis. $\mathrm{P}<0.05$ was considered to indicate a statistically significant difference.

\section{Results}

Histological changes. Hematoxylin and eosin-stained sections of aorta were examined for signs of atheroma (Fig. 2). The

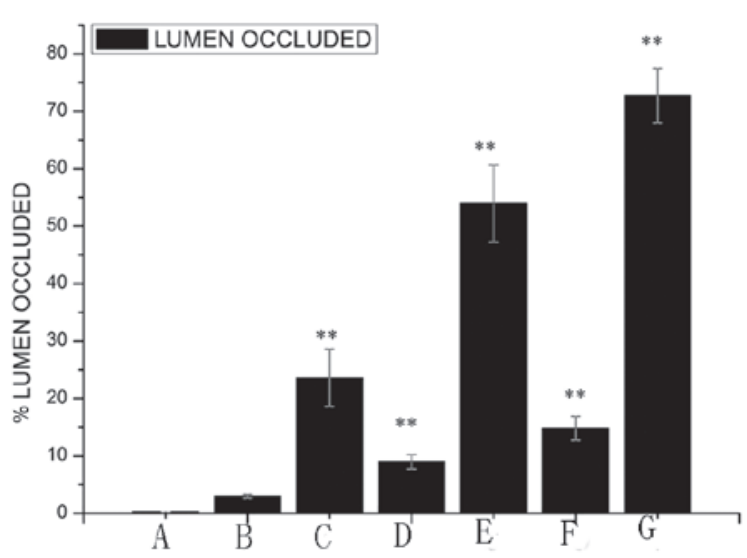

Figure 3. Occlusion of the lumen of the artery. (A) Control group; (B) high-fat diet 6-week group; (C) high-fat diet 8-week group; (D) high-fat diet 10-week group; (E) high-fat diet + balloon-injury 6-week group; (F) high-fat diet + balloon-injury 8 -week group and $(\mathrm{G})$ high-fat diet + balloon-injury 10 -week group. ${ }^{*} \mathrm{P}<0.05,{ }^{* *} \mathrm{P}<0.01$.

femoral artery of the control group had the following features: complete intimal endothelial cells arranged in neat rows; clear and intact internal elastic membrane; medial smooth muscle cell volume, size and arrangement were normal; and there was no inflammatory cell infiltration and lipid accumulation. Arterial lesions of the high-fat diet group show that the intimal membrane is composed mainly of foam cells, a small amount of inflammatory cell aggregation and no marked cap. In the high-fat diet + balloon-injury 6-week group, there was a significant fibrous cap. The fibrous cap had a few lipids, foam cells and other cell debris. In the high-fat diet + balloon-injury 10 -week group it was shown that the fibrous cap contained smooth muscle cells, foam cells and a lipid core in the intima, in addition to atherosclerotic lesions. With the development of lesions, the fibrous cap of the atherosclerotic material was gradually increased.

The percentage of vessel wall lumen occlusion was calculated (Fig. 3). The results showed that there was no marked occlusion of the lumen $(0.30 \pm 0.03 \%$, mean \pm SD) in the control group. There was a significant difference between the control group and the high-fat diet 6-week group $(1.98 \pm 0.34 \%$, $\mathrm{P}<0.05)$, high-fat diet 8 -week group $(8.97 \pm 1.2 \%, \mathrm{P}<0.01)$ and high-fat diet 10 -week group $(14.82 \pm 2.09 \%, \mathrm{P}<0.01)$. 


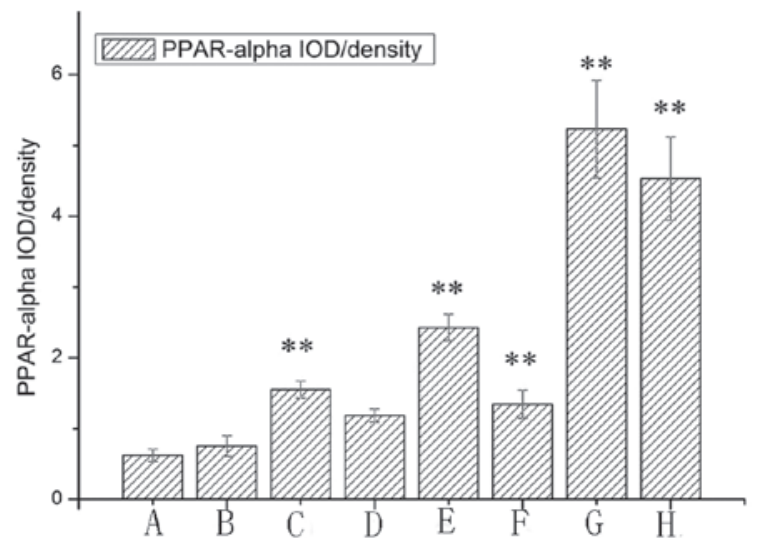

Figure 4. PPAR- $\alpha$ protein immunohistochemical staining in the vasculature. (A) Control group; (B) high-fat diet 6-week group; (C) high-fat diet 8-week group; (D) high-fat diet 10-week group; (E) high-fat diet+balloon-injury 6-week group; (F) high-fat diet+balloon-injury 8-week group; (G) high-fat diet+balloon-injury 10 -week lumen occluded $\geq 60 \%$ group and $(\mathrm{H})$ high-fat diet+balloon-injury 10 -week lumen occluded $<60 \%$ group. ${ }^{*} \mathrm{P}<0.05,{ }^{* *} \mathrm{P}<0.01$. PPAR- $\alpha$, peroxisome proliferator-activated receptor $\alpha$

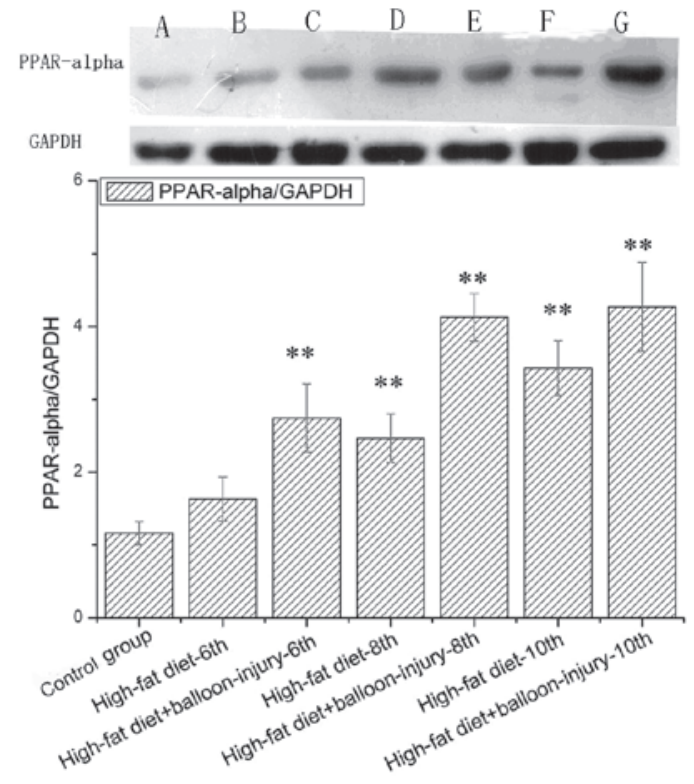

Figure 5. Protein products of PPAR- $\alpha$ and GPDH extracted from the vasculature: lane A, control group; lane B, high-fat diet 6-week group; lane $\mathrm{C}$, high-fat diet 8-week group; lane $\mathrm{D}$, high-fat diet 10-week group; lane E, high-fat diet+balloon-injury 6-week group; lane F, high-fat diet+ballooninjury 8-week group and lane $\mathrm{G}$, high-fat diet+balloon-injury 10-week group ${ }^{*} \mathrm{P}<0.05,{ }^{* *} \mathrm{P}<0.01$. PPAR- $\alpha$, peroxisome proliferator-activated receptor $\alpha$.

In the high-fat diet 10 -week group, the percentage of vessel wall lumen occlusion was higher than in the 6-week group. Compared with the control group, the percentage of vessel wall lumen occlusion in the high-fat diet + balloon-injury group was markedly increased $(\mathrm{P}<0.01)$. The high-fat diet + ballooninjury 6-group had $>23.62 \pm 4.97 \%$ occlusion of the lumen, the high-fat diet + balloon-injury 8-week group had $>53.97 \pm 6.7 \%$ occlusion of the lumen and the high-fat diet + balloon-injury 10 -week group had $>72.73 \pm 4.7 \%$ occlusion of the lumen. There was a significant difference between the various timepoint groups $(\mathrm{P}<0.01)$.

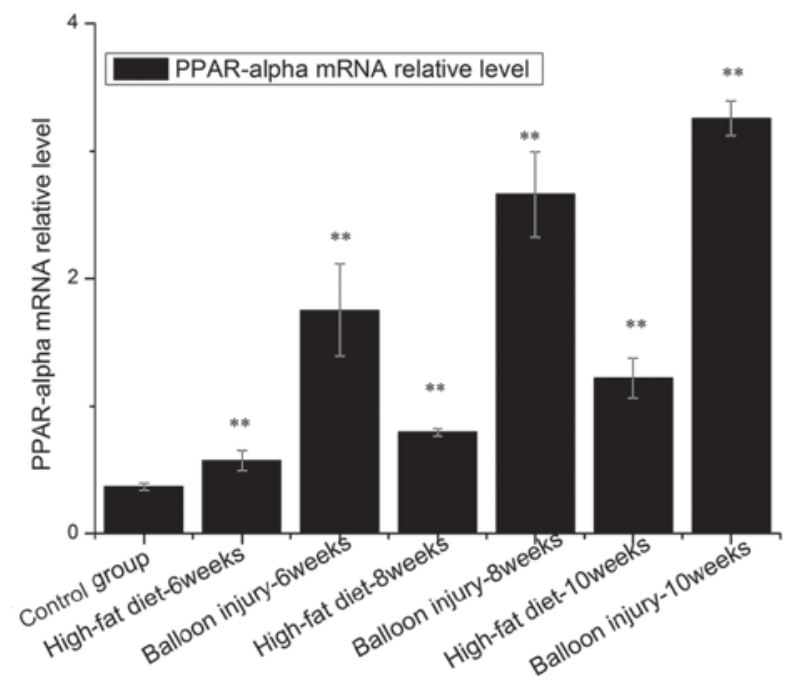

Figure 6. Gene expression of PPAR- $\alpha /$ GAPDH mRNA extracted from the vasculature. ${ }^{*} \mathrm{P}<0.05,{ }^{* *} \mathrm{P}<0.01$. PPAR- $\alpha$, peroxisome proliferator-activated receptor $\alpha$.

Location and expression of PPAR- $\alpha$ protein. IHC showed that PPAR- $\alpha$ with high staining intensity was seldom detected in the cytoplasm of the control group (Figs. 2 and 4). PPAR- $\alpha$ was detected at high staining intensities in the majority of lesions. Positive staining of PPAR- $\alpha$ was located in the cytoplasm of macrophages near the intima. In the high-fat diet and high-fat diet + balloon-injury groups, PPAR- $\alpha$ was higher than in the control group $(\mathrm{P}<0.01)$, with the exception of the high-fat diet 6 -week group $(\mathrm{P}>0.05)$. In the high-fat diet group, PPAR- $\alpha$ increased significantly from weeks 6 to $8(\mathrm{P}<0.01)$. In the high-fat diet group, from weeks 8 to 10 , PPAR- $\alpha$ increased markedly $(\mathrm{P}<0.05)$. In the high-fat diet + balloon-injury group, from weeks 6 to 10 , PPAR- $\alpha$ increased significantly $(\mathrm{P}<0.01)$.

According to the degree of occlusion, we divided the highfat diet + balloon-injury 10 -week group into $<60$ and $\geq 60 \%$ groups. In the $<60 \%$ group, PPAR- $\alpha$ expression was higher than in the $\geq 60 \%$ group $(\mathrm{P}<0.05)$.

Expression of PPAR- $\alpha$ protein. Western blotting was used to determine whether the PPAR- $\alpha$ protein level correlated with changes in its mRNA levels (Fig. 5). There was no difference between the control group and the 6-week high-fat diet group $(\mathrm{P}>0.05)$. In the high-fat diet group, PPAR- $\alpha$ expression was higher than the control group at the 8- and 10-week points $(\mathrm{P}<0.01)$. Furthermore, in the high-fat diet + balloon injury group, PPAR- $\alpha$ protein was expressed at significantly higher levels than in the control group. At the 10-week point, PPAR- $\alpha$ was 3.6 times more highly expressed compared with control group. From the 6 -week point to the 8 -week point, PPAR- $\alpha$ expression increased by $50.39 \%$. No such change was observed from weeks 8 to $10(\mathrm{P}>0.05)$.

Expression of PPAR- $\alpha$ gene. The expression level of PPAR- $\alpha$ mRNA and protein tended to increase in proportion to the severity of the lesion type (Fig. 6). Compared with the control group, PPAR- $\alpha$ mRNA was significantly increased in the high-fat diet or balloon injury groups $(\mathrm{P}<0.01)$. In the high-fat diet group, the increased level of PPAR- $\alpha$ was 7.18-fold higher 

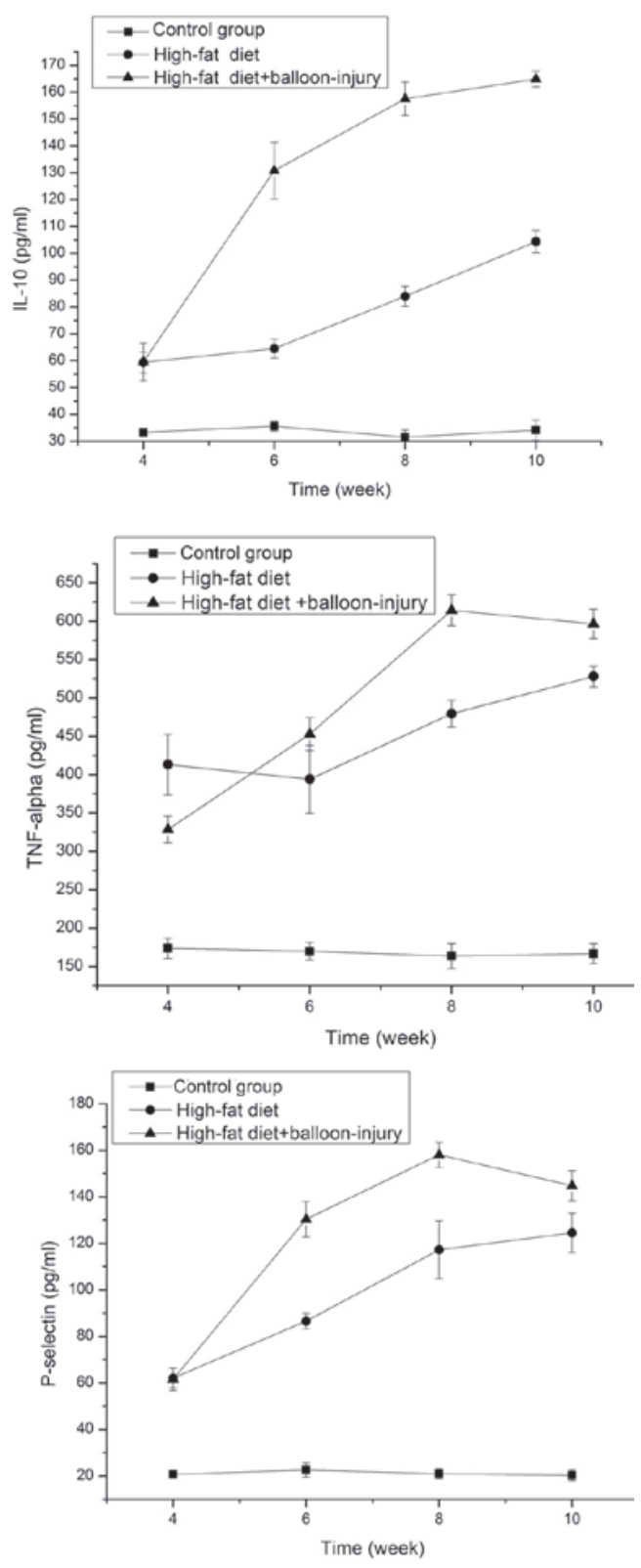

Figure 7. Levels of IL-10, TNF- $\alpha$ and P-selectin in the serum from weeks 4 to 10. IL, interleukin; TNF, tumor necrosis factor.

after rabbits were administered a $2 \%$ cholesterol diet for 6 weeks. As shown in Fig. 6, in the high-fat diet + ballooninjury group, the expression of PPAR- $\alpha$ was increased by 8.7-fold at week 10. Moreover, in the high-fat diet group, PPAR- $\alpha$ mRNA was more highly elevated at week 8 than at week $6(\mathrm{P}<0.01)$. PPAR- $\alpha$ mRNA at week 10 was higher than at week $8(\mathrm{P}<0.01)$. In the high-fat diet + balloon-injury group, we also observed that PPAR- $\alpha$ was increased significantly from weeks 6 to $10(\mathrm{P}<0.01)$, while this change was larger than in the high-fat diet group $(\mathrm{P}<0.01)$.

IL-10 in the serum. In the high-fat diet and high-fat diet + balloon-injury groups, IL-10 levels were higher than in the control group $(\mathrm{P}<0.01)$. In the high-fat diet group, IL-10 was higher at week 4 than at week $6(\mathrm{P}<0.01)$. In addition, there was marked growth from weeks 6 to $8(\mathrm{P}<0.01)$, as well as from weeks 8 to $10(\mathrm{P}<0.01)$. IL-10 increased to 3.31 times the control group level at week 10. In the balloon-injury group, the expression level of IL-10 was also significantly higher than in the control group $(\mathrm{P}<0.01)$. From weeks 4 to 8 , there was a significant difference $(\mathrm{P}<0.01)$. However, the growth rate became slower between the high-fat diet + balloon-injury 8 -week group and the high-fat diet + balloon-injury 10 -week group $(\mathrm{P}<0.05)$. At 10 weeks, IL-10 was 5.25 times higher compared to the control group (Fig. 7).

$T N F-\alpha$ in the serum. In the high-fat diet and high-fat diet + balloon-injury groups, TNF- $\alpha$ levels were higher than in the control group $(\mathrm{P}<0.01)$. The expression of TNF- $\alpha$ showed no marked difference between weeks 4 and 6 in the high-fat diet group $(P>0.05)$. From weeks 6 to 8 , the level of TNF- $\alpha$ showed a significant difference $(\mathrm{P}<0.01)$. In the high-fat diet 10 -week group, TNF- $\alpha$ was increased by 3.23 -fold compared with the control group. From weeks 4 to 8 , the expression of TNF- $\alpha$ was increased in the high-fat diet + balloon-injury group $(\mathrm{P}<0.01)$. From weeks 8 to 10 , the level of TNF- $\alpha$ was decreased $(\mathrm{P}>0.05)$. At the 8 -week time point, TNF- $\alpha$ was increased by 3.75 times compared with the control group (Fig. 7).

P-selectin in the serum. In the high-fat diet and high-fat diet + balloon-injury groups, P-selectin levels were higher than in the control group $(\mathrm{P}<0.01)$. In the high-fat diet group, the level of P-selectin was significantly different between weeks 4 and $8(\mathrm{P}<0.01)$. However, the rate of growth decreased between the high-fat diet 8 -week and high-fat diet 10 -week groups $(\mathrm{P}<0.05)$. In the high-fat diet 10 -week group, $\mathrm{P}$-selectin was 6.13 -fold higher than in the control group. In the high-fat diet + balloon-injury group, there was a significant difference between weeks 4 and $8(\mathrm{P}<0.01)$. However, P-selectin was lower in the high-fat diet + balloon-injury 10-week group than in the high-fat diet + balloon-injury 8 -week group $(\mathrm{P}<0.05)$. $\mathrm{P}$-selectin was 7.78-fold higher than the control group in the high-fat diet + balloon-injury 8-week group (Fig. 7).

\section{Discussion}

Due to atherosclerosis, cardiovascular disease is currently the major cause of disability and mortality. Therefore, investigating the etiopathogenesis and pathogenicity of atherosclerosis and taking effective measures to delay and reverse the progression of atherosclerosis have become important topics of study. However, atherosclerosis has a complex multifactorial pathophysiology and a number of risk factors work together to shape the formation of plaques. Activated PPAR- $\alpha$ is important in the regulation of cellular development and differentiation and the metabolism of body fuel including carbohydrates, lipids and proteins $(13,14)$. Although PPAR- $\alpha$ has been demonstrated to be involved in atherosclerosis, the potential mechanisms of PPAR- $\alpha$ remain to be determined. The present study was undertaken in order to test our hypothesis that PPAR- $\alpha$ activity may have vascular protective effects in atherosclerosis. The major finding of our present study was that PPAR- $\alpha$ activation played a role in atherosclerosis, leading to partial inhibition of the development of lesions. We further found that PPAR- $\alpha$ influenced atherosclerosis via decreasing inflammation and P-selectin. 
Collectively, our present observations highlight PPAR- $\alpha$ as a promising therapeutic agent for the treatment of vascular injury in a high-fat diet and balloon-injury model.

A previous study showed that lipolytic products are capable of activating PPAR- $\alpha$ in brown adipocytes, thereby expanding the oxidative capacity in order to match enhanced fatty acid supply (15). Furthermore, activation of PPAR- $\alpha$ has a critical role in controlling the cholesterol cycle in macrophages. Scavenger receptor class B type I (SR-B I), which plays a role in cholesterol efflux from macrophages, is upregulated by PPAR- $\alpha$ ligands in macrophages, leading to increased lipolysis (16). These collective effects of PPAR- $\alpha$ should enhance transport of cholesterol from peripheral tissues to the liver and promote proatherogenic responses. In this study, we demonstrated that PPAR- $\alpha$ mRNA and protein were significantly increased in high-fat diet or highfat diet + balloon-injury groups $(\mathrm{P}<0.01)$, as compared with the control group. Furthermore, the expression of PPAR- $\alpha$ was significantly higher in the high-fat diet + balloon-injury group than in the high-fat diet group at the same time point. In the immunohistochemical experiment, our results revealed that a high expression of PPAR- $\alpha$ occurred in macrophages of the high-fat diet and high-fat diet + balloon-injury groups, particularly in the plaque shoulder, but the expression of PPAR- $\alpha$ was low in the control group. Notably, in the high-fat diet + balloon-injury 10 -week group we found that in the $<60 \%$ group, PPAR- $\alpha$ was higher than in the $\geq 60 \%$ group $(\mathrm{P}<0.01)$. In addition, Sueyoshi et al (17) showed that PPAR- $\alpha$ mRNA increased significantly in atherosclerosis and tended to increase in proportion to the severity of the lesion, which is in line with the findings of the present study. In our model, high triglyceride load may cause PPAR- $\alpha$ activation. There is abundant evidence that PPAR- $\alpha$ inhibited extracellular matrix metalloprotease activity (18) and foam cell formation (19). Our results suggest that PPAR- $\alpha$ is involved in the chain of events leading to atherosclerosis in a protective manner, and that combined therapy including PPAR- $\alpha$ agonists may have a synergistic effect on inhibiting atherosclerosis.

A complex network of inflammatory cytokines and chemokines plays a major role in mediating, amplifying and perpetuating the atherosclerosis process (20). Among pro- and anti-inflammatory cytokines, TNF- $\alpha$ and IL-10 play critical roles in the formation of atherosclerosis (21). As an anti-inflammatory cytokine, IL-10 in atherosclerotic plaques in mice and in the peripheral circulation of patients was higher (22). Previous studies on certain other inflammatory diseases, such as Alzheimer's disease (AD) and Crohn's disease, found that PPAR- $\alpha$ and IL-10 have significant interactions $(23,24)$. On the contrary, TNF- $\alpha$ is an important proinflammatory cytokine that may stimulate production of a host of other cytokines (25). Decreased TNF- $\alpha$ significantly improves endothelial and adipose tissue dysfunction in pre-diabetic patients with coronary artery disease (CAD) (26). The levels of these cytokines were assessed in our study. The analyses demonstrated that the concentration of IL-10 and TNF- $\alpha$ significantly increased in high-fat diet and high-fat diet + balloon-injury groups $(\mathrm{P}<0.01)$. Notably, the level of IL-10 increased between the 8th and 10th week in the high-fat diet + balloon-injury group, while the level of TNF- $\alpha$ decreased. Based on our results and other known characteristics of PPAR- $\alpha$, we hypothesize that IL-10 activation may occur with atherosclerosis, which may explain some of the beneficial effects of PPAR- $\alpha$ on vascular cells. PPAR- $\alpha$ may cause this anti-inflammatory effect through TLR, MAPK and NF-KB pathways (27-30).

The absence of PPAR- $\alpha$ is capable of upregulating P-selectin in acute pancreatitis induced by cerulean (31). However, the role of PPAR- $\alpha$ on P-selectin in atherosclerosis remains to be elucidated. The inflammatory response is the main process by which inflammatory factors are involved in atherosclerotic lesions. During the occurrence and development of atherosclerotic lesions, lipid accumulation plays an important role which results from inflammatory cells ingratiating. P-selectin is involved in these two stages (32). There are few data available on P-selectin and artery atherosclerosis. Our experiments found that P-selectin levels were lower in the high-fat diet + ballooninjury 10-week group than in the high-fat diet + balloon-injury 8 -week group $(\mathrm{P}<0.05)$. Anti-P-selectin therapy may be a powerful tool in inhibiting atherosclerotic lesion progression.

In conclusion, we demonstrated that PPAR- $\alpha$ was associated with atheromatous plaque formation in the hypercholesterolemic and balloon-injury model. PPAR- $\alpha$ expression was an adaptive response that results in a slow progression of atherosclerosis, although vascular occlusions were serious in the high-fat diet + balloon-injury group. If the experimental time or treatment with PPAR- $\alpha$ agonists is extended, the lesions may become smaller. We further observed that PPAR- $\alpha$ protected against atherosclerosis by increasing IL-10, suppressing TNF- $\alpha$ and regulating $\mathrm{P}$-selectin, although a detailed analysis of the molecular mechanisms in atherosclerosis is required in order to increase understanding. Previous studies have reported beneficial effects of PPAR- $\alpha$ agonists such as fibrates, bezafibrate and WY14643 $(33,34)$. These results suggest that PPAR- $\alpha$ agonists can be expected to protect against the progression of atherosclerosis in hyperlipidemic patients.

\section{Acknowledgements}

The study was supported by the Shanghai Rising-Star Program (08QA1404100 grant) and the National Natural Science Foundation of China (30971265).

\section{References}

1. Libby P, Ridker PM and Hansson GK: Progress and challenges in translating the biology of atherosclerosis. Nature 473: 317-325, 2011.

2. Medh JD: Peroxisome proliferator-activated receptors in atherosclerosis. Curr Opin Lipidol 10: 69-71, 1999.

3. Oyekan A: PPARs and their effects on the cardiovascular system. Clin Exp Hypertens 33: 287-293, 2011.

4. Shah A, RaderDJ and Millar JS: The effect of PPAR-alpha agonism on apolipoprotein metabolism in humans. Atherosclerosis 210: 35-40, 2010.

5. Kraja AT, Province MA, Straka RJ, Ordovas JM, Borecki IB and Arnett DK: Fenofibrate and metabolic syndrome. Endocr Metab Immune Disord Drug Targets 10: 138-148, 2010.

6. Hashizume S, Akaike M, Azuma $\mathrm{H}$, et al: Activation of peroxisome proliferator-activated receptor $\alpha$ in megakaryocytes reduces platelet-derived growth factor-BB in platelets. J Atheroscler Thromb 18: 138-147, 2011.

7. Wagner JD, Shadoan MK, Zhang L, et al: A selective peroxisome proliferator-activated receptor alpha agonist, CP-900691, improves plasma lipids, lipoproteins, and glycemic control in diabetic monkeys. J Pharmacol Exp Ther 333: 844-853, 2010. 
8. Marx N, Sukhova GK, Collins T, Libby P and Plutzky J: PPARalpha activators inhibit cytokine-induced vascular cell adhesion molecule-1 expression in human endothelial cells. Circulation 99: 3125-3131, 1999.

9. Mueller M, Hobiger S and Jungbauer A: Red clover extract: a source for substances that activate peroxisome proliferatoractivated receptor alpha and ameliorate the cytokine secretion profile of lipopolysaccharide-stimulated macrophages. Menopause 17: 379-387, 2010.

10. WoffordJL,KahlFR,HowardGR,McKinneyWM,TooleJand Crouse JR III: Relation of extent of extracranial carotid artery atherosclerosis as measured by B-mode ultrasound to the extent of coronary atherosclerosis. Arterioscler Thromb 11: 1786-1794, 1991.

11. Buchanan MR and Brister SJ: Inhibition of chronic vessel wal intimal hyperplasia following acute anticoagulant treatment: relative effects of heparin and dermatan sulphate. Thromb Res 91: 157-167, 1998

12. Jia XL, Li SY, Dang SS, et al: Increased expression of chondroitin sulphate proteoglycans in rat hepatocellular carcinoma tissues. World J Gastroenterol: 18: 3962-3976, 2012.

13. Campioli E, Batarseh A, Li J and Papadopoulos V: The endocrine disruptor mono-(2-ethylhexyl) phthalate affects the differentiation of human liposarcoma cells (SW 872). PLoS One 6: e28750, 2011.

14. Soymorphin-5, a soy-derived $\mu$-opioid peptide, decreases glucose and triglyceride levels through activating adiponectin and PPARo systems in diabetic KKAy mice. Am J Physiol Endocrinol Metab 302: E433-E440, 2012.

15. Mottillo EP, Bloch AE, Leff T and Granneman JG: Lipolytic products activate peroxisome proliferator-activated receptor (PPAR) $\alpha$ and $\delta$ in brown adipocytes to match fatty acid oxidation with supply. J Biol Chem 287: 25038-25048, 2012.

16. Chinetti G, Gbaguidi FG, Griglio S, et al: CLA-1/SR-BI is expressed in atherosclerotic lesion macrophages and regulated by activators of peroxisome proliferator-activated receptors. Circulation 101: 2411-2417, 2000.

17. Sueyoshi S, Mitsumata M, Kusumi Y, et al: Increased expression of peroxisome proliferator-activated receptor (PPAR)-alpha and PPAR-gamma in human atherosclerosis. Pathol Res Pract 206 429-438, 2010

18. Duhaney TAS, Cui L, Rude MK, et al: Peroxisome proliferatoractivated receptor alpha-independent actions of fenofibrate exacerbates left ventricular dilation and fibrosis in chronic pressure overload. Hypertension 49: 1084-1094, 2007.

19. Dushkin M, Khoshchenko O, Posokhova E and Schvarts YS Agonists of PPAR-alpha, PPAR-gamma, and RXR inhibit the formation of foam cells from macrophages in mice with inflammation. Bull Exp Biol Med 144: 713-716, 2007.

20. Stoll G and Bendszus M: Inflammation and atherosclerosis: novel insights into plaque formation and destabilization. Stroke 37: 1923-1932, 2006.
21. Kabłak A, Ziembicka TP, Stẹpień E, et al: Relationship between carotid intima-media thickness, cytokines, atherosclerosis extent and a two-year cardiovascular risk in patients with arteriosclerosis. Kardiol Pol 69: 1024-1031, 2011.

22. Meng X, Zhang K, Li J, et al: Statins induce the accumulation of regulatory $\mathrm{T}$ cells in atherosclerotic plaque. Mol Med 18: 598-605, 2012.

23. Heun R, Kölsch H, Ibrahim-Verbaas CA, et al: Interactions between PPAR- $\alpha$ and inflammation-related cytokine genes on the development of Alzheimer's disease, observed by the Epistasis Project. Int J Mol Epidemiol Genet 3: 39-47, 2012.

24. Lee JW, Bajwa PJ, Carson MJ, et al: Fenofibrate represses interleukin-17 and interferon-gamma expression and improves colitis in interleukin-10-deficient mice. Gastroenterology 133: 108-123, 2007.

25. Donnelly SC, Strieter RM, Reid PT, et al: The association between mortality rates and decreased concentrations of interleukin-10 and interleukin-1 receptor antagonist in the lung fluids of patients with the adult respiratory distress syndrome. Ann Intern Med 125: 191-196, 1996.

26. Rizza S, Cardellini M, Porzio O, et al: Pioglitazone improves endothelial and adipose tissue dysfunction in pre-diabetic CAD subjects. Atherosclerosis 215: 180-183, 2011.

27. Parameswaran $\mathrm{N}$ and Patial S: Tumor necrosis factor- $\alpha$ signaling in macrophages. Crit Rev Eukaryot Gene Expr 20: 87-103, 2010.

28. Hou X, Shen YH, Li C, et al: PPARalpha agonist fenofibrate protects the kidney from hypertensive injury in spontaneously hypertensive rats via inhibition of oxidative stress and MAPK activity. Biochem Biophys Res Commun 394: 653-659, 2010.

29. Rinaldi B, Donniacuo M, Esposito E, et al: PPAR $\alpha$ mediates the anti-inflammatory effect of simvastatin in an experimental model of zymosan-induced multiple organ failure. Br J Pharmacol 163: 609-623, 2011

30. Matta R, Barnard JA, Wancket LM, et al: Knockout of Mkp-1 exacerbates colitis in Il-10-deficient mice. Am J Physiol Gastrointest Liver Physiol 302: G1322-G1335, 2012.

31. Genovese T, Mazzon E, Di Paola R, et al: Role of peroxisome proliferator-activated receptor-alpha in acute pancreatitis induced by cerulein. Immunology 118: 559-570, 2006.

32. Johnson RC, Chapman SM, Dong ZM, et al: Absence of P-selectin delays fatty streak formation in mice. J Clin Invest 99: 1037-1043, 1997.

33. Koh KK, Han SH, Quon MJ, Yeal Ahn J and Shin EK: Beneficial effects of fenofibrate to improve endothelial dysfunction and raise adiponectin levels in patients with primary hypertriglyceridemia. Diabetes Care 28: 1419-1424, 2005.

34. Nakamachi T, Nomiyama T, Gizard F, et al: PPARalpha agonists suppress osteopontin expression in macrophages and decrease plasma levels in patients with type 2 diabetes. Diabetes 56 : 1662-1670, 2007 